\title{
THIS MONTH IN NATURE BIOTECHNOLOGY
}

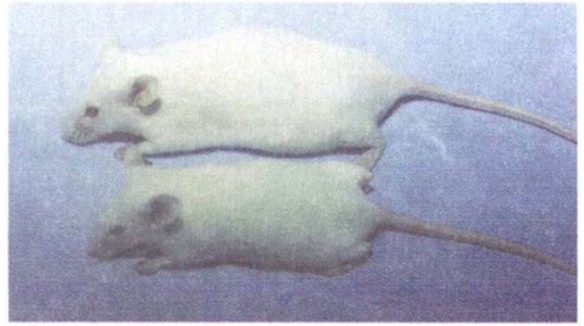

By engineering mice that can be induced to express a transgene with subphysiological levels of the progesterone antagonist RU 486 in a tissue-specific manner (see pp. 219 and 239), human growth hormone has been shown to stimulate growth when expressed for a limited time in the liver of bigenic mice.

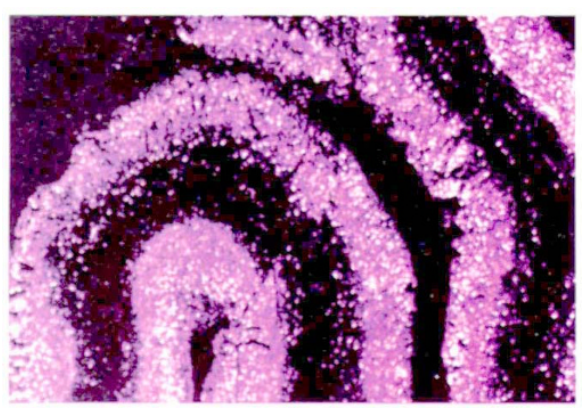

Senile plaques-aggregates of insoluble $\beta$ amyloid peptides (A $\beta$ )-are thought to underlie the degenerative processes leading to Alzheimer's disease and are thus an important therapeutic drug target. As an alternative to the use of brain sections for screening, a synthetic template (synthaloid) composed of fibrillar $A \beta$ aggregates immobilized in a polymer matrix has been developed to mimic $\mathbf{A} \beta$ deposition and thus allow identification of inhibitors under physiological conditions (see pp. 223 and 258).

\section{Organic solvents detect protein-binding sites}

Small organic molecules, in combination with X-ray crystallography, have been used as probes for mapping surface binding sites on proteins. Using hen egg-white lysozyme (HEWL) as a model protein, Edvards Liepinsh and Gottfried Otting (Karolinska Institute, Stockholm, Sweden) have shown that NMR can be used to detect magnetization transfer between the protons of the protein in the active cleft and small organic ligands to determine binding sites (see p. 264). This method of modeling protein-ligand interactions in solution offers an alternative to crystal modeling with potential for structural analysis for drug design.

Research briefs written by Emma Johnson.
A potentially inexpensive and safe way to produce animal vaccines is to infect plants with chimeric virus particles (CVP) expressing immunogenic viral epitopes. The effectiveness of this strategy has been demonstrated by Dalsgaard and coworkers, who have engineered a mink enteritis viral epitope into cowpea mosaic virus and propagated the CVPs in black-eyed bean (see pp. 221 and 248). A single subcutaneous injection conferred dose-dependent protection against enteritis in mink. Higher doses of CVP abolished shedding of the virus in feces, an important proof of the vaccine's effectiveness, as most enteritis infections occur via oral-fecal transmission.

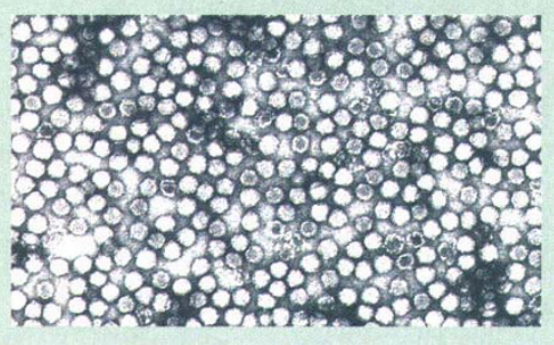

\section{Single-step wine fermentation}

Alcoholic fermentation in wine-making is often followed by a malolactic fermentation process to reduce the acidity of the wine. Separate fermentations are necessary, as the wine yeast, Saccharomyces cerevisiae, does not contain the enzymes necessary to degrade organic acids such as tartaric and malic acid. This extrabacterial fermentation, or chemical neutralization, introduces another level of complexity to the art of wine making. By introducing both a malolactic enzyme as well as a permease, a functional malolactic strain of $S$. cerevisiae has been engineered (see pp. 224 and 253). In certain wines, the engineered strain may reduce spoilage and thus enhance flavor.

\section{Reversible cell membrane permeabilization}

Membrane-permeabilizing methods have aided the study of small molecules in the intracellular environment of living cells. Irreversible treatments with electroporation or detergents or bacterial toxins often lead to cell damage and death. To permeabilize cell membranes in a reversible manner, Mehmet Toner and colleagues have engineered a Staphylococcus $\alpha$-exotoxin to produce transmembrane pores that close in a dose-dependent response to $\mathrm{Zn}^{2+}$ (see p. 278). A $\mathrm{Zn}^{2+}$-chelating agent can subsequently be added to open the pore.
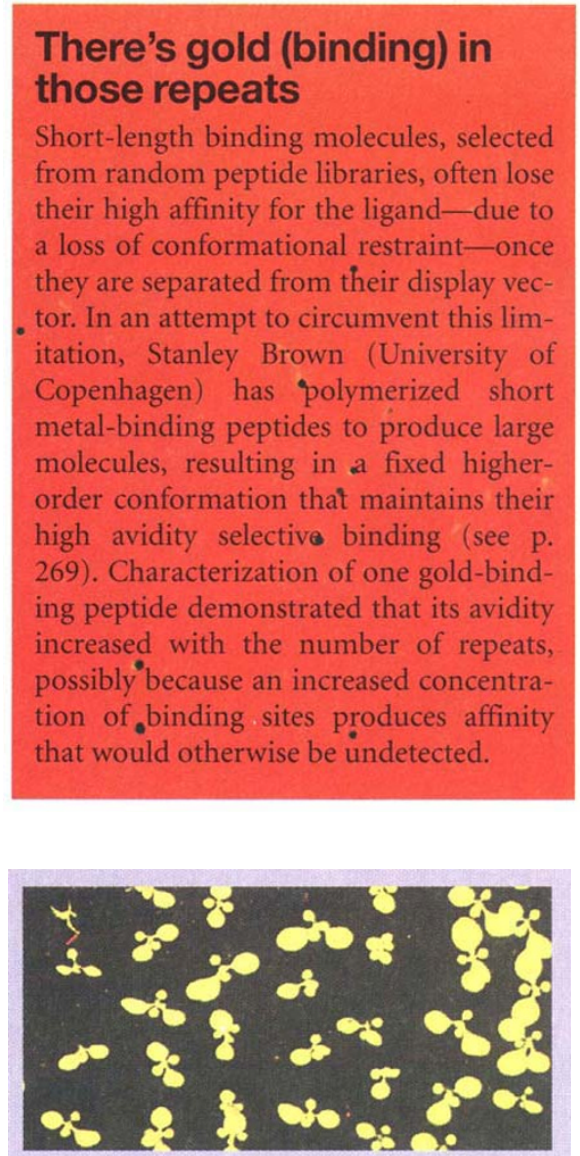

When the Vitreoscilla hemoglobin (VHb) is expressed in $E$. coli, the enhanced oxygen metabolism that results is reflected in increased growth. To determine the effects of the expression of this oxygen-binding protein on plant growth, the same gene has been used to transform tobacco (see pp. 222 and 248). Expression of heterologous vhb had farreaching effects on the plants' metabolism, increasing dry weight yield in transgenics by $80-100 \%$, compared with wild-type plants, altering metabolite production, and reducing flowering and germination times.

\section{DNA nanocircles roll along}

Small catalytic RNAs are usually synthesized by either in vitro transcription runoff or stepwise chemical synthesis on solid supports. Sarah Daubendiek and Eric Kool (University of Rochester, NY) have simplified matters by designing promoterless, single-stranded DNA circles, encoding a hammerhead ribozyme, that can be transcribed in vitro by several different RNA polymerases (see p. 273). The resulting concatomeric RNAs ultimately self-cleave to form active monomers that maintain their ability to cleave RNAs in a sequence-specific manner in trans. 